\title{
Original
}

\section{Prognostic Factors for Recurrence after Tegafur-uracil Plus Leucovorin Adjuvant Chemotherapy in Patients with Colorectal Cancer}

\author{
Makoto Watanabe*, Masahiko Murakami, Yoshiaki Ozawa, \\ Satoru Goto, Akira Fujimori, Koji OtsukA \\ and Takeshi AokI
}

\begin{abstract}
To evaluate prognostic factors for recurrence after tegafur-uracil plus leucovorin (UFT/LV) adjuvant chemotherapy in patients with colorectal cancer (CRC). Consecutive patients with CRC who received UFT/LV as adjuvant chemotherapy at Showa University Hospital between June 2005 and December 2008 were included in the study, 5-year disease-free survival (DFS) and overall survival (OS) rates were estimated, and prognostic factors for recurrence were analyzed using the Cox proportional hazards model for multivariate analysis. Of 92 patients included in the study, $17(18.5 \%)$ had disease recurrence. The 5-year DFS and OS rates were $82.2 \%$ and $91.9 \%$, respectively. In the multivariate analysis, preoperative CA19-9 level > $>37 \mathrm{U} / \mathrm{ml}$, emergency operation, and T4 lesions were independent significant prognostic factors after treatment with UFT/LV adjuvant chemotherapy. The three independent prognostic factors - T4 lesions, emergency operation, and high preoperative CA19-9 levels - may be useful for decision-making regarding whether patients should receive 5-fluouracil-based or L-oxaliplatin-based adjuvant chemotherapy. As this was a single-institution study with a small number of patients, our findings need to be confirmed in larger multicenter studies..
\end{abstract}

Key words : adjuvant chemotherapy, colorectal cancer, UFT / LV, long-term outcomes, prognostic factors

\section{Introduction}

In patients with cancer, postoperative adjuvant chemotherapy reduces disease recurrence and improves overall survival. Such benefits for patients with Stage III colorectal cancer (CRC) are great enough to recommend adjuvant chemotherapy ${ }^{1)}$. Based on the results from the Japan Clinical Oncology Group (JCOG0205) ${ }^{2)}$ and the National Surgical Adjuvant Breast and Bowel Protocol (NSABP C-06) ${ }^{3}$, a regimen of uracil and tegafur plus leucovorin (UFT/LV) has been widely used as standard postoperative adjuvant chemotherapy for Stage III CRC in Japan.

L-oxaliplatin (L-OHP)-based adjuvant chemotherapy such as FOLFOX (oxaliplatin plus infusional leucovorin and fluorouracil $)^{4)}$ and 5-fluouracil (5-FU)-based adjuvant chemotherapy such as capcitabine ${ }^{5)}$ or 5-FU / LV are also used as standard adjuvant chemotherapy for Stage III

Department of Surgery, Division of General and Gastroenterological Surgery, Showa University School of Medicine, 1-5-8 Hatanodai, Shinagawa-ku, Tokyo 142-8666 Japan.

* To whom corresponding should be addressed. 
CRC in Japan ${ }^{6)}$. Furthermore, based on the results of the Adjuvant Chemotherapy Trial of TS-1 for Colon Cancer (ACTS-CC) trial ${ }^{7)}$, which demonstrated non-inferiority of S-1 compared with UFT / LV for Stage III colon cancer, S-1 will also be a new adjuvant chemotherapy option for colon cancer. However, clinically useful predictors that can help select adjuvant chemotherapy regimens have not been identified. Although various findings in patients with Stage II CRC, including poorly differentiated histology, T4 lesions, perforation, and inadequately sampled lymph nodes $(n<13)$, have been considered for use in selection of adjuvant chemotherapy ${ }^{8-10)}$, there is no international consensus. Therefore, we evaluated long-term outcomes and prognostic factors for recurrence after UFT/LV adjuvant chemotherapy in patients with Stage II or III CRC and identified potential selection criteria for UFT / LV as adjuvant chemotherapy.

\section{Materials and methods}

\section{Patients}

From June 2005 to December 2008, consecutive patients who received oral UFT/LV as adjuvant chemotherapy only for $\mathrm{CRC}$ at Showa University Hospital were prospectively enrolled. The main inclusion criteria for treatment with UFT / LV adjuvant chemotherapy were: histologically proven Stage II or III colorectal adenocarcinoma, an Eastern Cooperative Oncology Group performance status (PS) of $\leq 1$, no prior chemotherapy or radiotherapy for CRC, and adequate bone marrow, renal and hepatic function.

This study was conducted in accordance with the Declaration of Helsinki. Written informed consent was obtained from all patients, with the approval of the ethics committee of Showa University Hospital.

\section{Treatment protocol}

We administered chemotherapy to all eligible patients between 3 and 6 weeks after surgery. UFT (300 mg / $\mathrm{m}^{2} /$ day) and LV (75 mg/body/day) were administered orally on days 1-28, followed by a 7-day rest period; this 35-day cycle was repeated up to five times. The daily UFT and LV doses were divided into three doses that were given $8 \mathrm{~h}$ apart with water. Patients were instructed to avoid consuming food during the hour before and the hour after each dose. Additional details and toxicity assessments have been described elsewhere ${ }^{11)}$.

\section{Patient follow-up and recurrence of disease}

After completing chemotherapy, patients were scheduled for follow-up as outpatients every 3 months during the first 3 years, every 6 months during the next 2 years, and annually thereafter, as per the 2010 Japanese Society for Cancer of the Colon and Rectum (JSCCR) guidelines ${ }^{6}$. Levels of serum tumor markers (carcinoembryonic antigen [CEA] and carbohydrate antigen 19-9 [CA19-9]) were measured every 3 months for the first 3 years and every 6 months for the next 2 years. Computed tomography (CT) scans of the chest, abdomen, and pelvis were performed every 6 months for 5 years. Colonoscopies were performed every 12 months for 3 years. 
Local recurrence was defined as clinical or radiologic tumor regrowth within the previous pelvic treatment field, peritoneum, or anastomosis. Distant recurrence was defined as tumor growth in any other area. Overall survival (OS) was calculated from the date of surgery to the date of death or last follow-up. Disease-free survival (DFS) was defined as the period from the date of surgery to the date of recurrence or last follow-up.

\section{Clinical and pathological variables}

The patient characteristics that we recorded included age, sex, PS, tumor location, preoperative CEA and CA19-9 levels, histologic type, tumor size, depth of tumor, lymphatic and venous invasion, number of lymph node (LN) metastases, degree of LN metastases, pathological stage, surgical approach and procedure, level of LN dissection, number of LNs examined, and postoperative complications. DFS, OS, and the relationship between clinicopathological variables and development of recurrence were analyzed. The level of LN dissection and histologic types were evaluated according to the Japanese Classification of Colorectal Carcinoma, Second English Edition ${ }^{12)}$. Depth of tumor, degree of LN metastases, and pathological stage were categorized according to the Seventh Edition of the TNM Classification (TNM7th) ${ }^{13)}$.

\section{Statistical analysis}

Continuous variables are expressed as medians and ranges. Categorical variables are expressed as numbers and percentages. The relationship between clinicopathological variables and development of recurrence were assessed by univariate analyses using Cox proportional hazards regression models and are expressed as hazard ratios and 95\% confidence intervals (CIs). All factors with a $p$ value of $<0.05$ were introduced into multivariate Cox regression analyses with a semi-manual backward (likelihood ratio) variable selection. The 5-year DFS and OS rates were estimated using the Kaplan-Meier method. Finally, based on the number of prognostic factors for recurrence, DFS and OS were compared using a log-rank test and Wilcoxon test. All statistical analyses were performed using $\mathrm{JMP}^{\circledR}$ Pro version 11.0.0 software (SAS Institute, Cary, $\mathrm{NC}$, USA) and $p$ values of $<0.05$ were considered statistically significant.

\section{Results}

\section{Patient characteristics and surgical outcomes}

A total of 92 patients were enrolled in the study. Patient characteristics and surgical outcomes are summarized in Table 1. Emergency operations were performed for two patients (2.2\%) due to tumor perforation.

\section{Evolution of disease}

The median follow-up period was 70 months (range, 9-120 months). Seventeen patients (18.5\%) developed disease recurrence; their characteristics are listed in Table 2. Eight of them $(47.1 \%$ ) had local recurrence (median time to relapse, 23.3 months) - three developed peritoneal recurrences, and five developed anastomotic recurrences. The other nine (52.9\%) 
Table 1. Patient characteristics and surgical outcomes $(\mathrm{N}=92)$

\begin{tabular}{|c|c|c|c|c|c|}
\hline Age (years) & Median (range) & $67(30-84)$ & Operation, N (\%) & Elective & $90(97.8)$ \\
\hline Sex & $\mathrm{M} / \mathrm{F}$ & $60 / 32$ & \multirow{2}{*}{ Surgical approach, N (\%) } & & $2 \quad(2.2)$ \\
\hline \multirow[t]{2}{*}{ PS (ECOG), N (\%) } & 0 & $70(76.1)$ & & Laparoscopic & $40(43.5)$ \\
\hline & 1 & $22(23.9)$ & \multirow{6}{*}{ Surgical procedure, N (\%) } & Laparotomy & $\begin{array}{ll}52 & (56.5) \\
57 & (620)\end{array}$ \\
\hline \multirow[t]{3}{*}{ Tumor location, N (\%) } & Right-side colon $(\mathrm{C}, \mathrm{A}, \mathrm{T})$ & $29(31.5)$ & & HAR & $\begin{array}{ll}31 & (0.0) \\
11 & (119)\end{array}$ \\
\hline & Left-side colon $(\mathrm{D}, \mathrm{S})$ & $27(29.3)$ & & LAR & $11(11.9)$ \\
\hline & Rectum & $36(39.1)$ & & uLAR & 7 (7.6) \\
\hline \multirow[t]{3}{*}{ Preoperative CEA level (ng/ml), N (\%) } & $\leq 5.1$ & $45(48.9)$ & & Hartmann & $1 \quad(1.1)$ \\
\hline & $>5.1$ & $32(34.8)$ & & APR & $5 \quad(5.4)$ \\
\hline & Unknown & $15(16.3)$ & \multirow[t]{3}{*}{ Level of LN dissectiona, $\mathrm{N}$ (\%) } & D1 & $2(2.2)$ \\
\hline \multirow[t]{3}{*}{ Preoperative CA19-9 level $(\mathrm{U} / \mathrm{ml}), \mathrm{N}(\%)$} & $\leq 37$ & $66(71.7)$ & & D2 & $36(39.1)$ \\
\hline & $>37$ & $9 \quad(9.8)$ & & D3 & $54(58.7)$ \\
\hline & Unknown & $17(18.5)$ & \multirow{5}{*}{$\begin{array}{l}\text { No. of LNs examined, }(\mathrm{N}) \\
\text { Operating time }(\mathrm{min}) \\
\text { Operative blood loss }(\mathrm{ml}) \\
\text { Complications, } \mathrm{N}(\%)\end{array}$} & Median (range) & $20(4-52)$ \\
\hline \multirow[t]{4}{*}{ Histologic types ${ }^{\mathrm{a}}, \mathrm{N}(\%)$} & Papillaly & $1 \quad(1.1)$ & & Median (range) & $200(95-735)$ \\
\hline & Tublar & $82(89.1)$ & & Median (range) & $120(3-1070)$ \\
\hline & poorly & $6 \quad(6.5)$ & & None & $70(76.1)$ \\
\hline & Mucinous & $3 \quad(3.3)$ & & Superficial SSI & $7 \quad(7.6)$ \\
\hline Tumor size $(\mathrm{mm})$ & Median (range) & $45(17-90)$ & & Organ / space SSI & $2 \quad(2.2)$ \\
\hline \multirow[t]{4}{*}{ Depth of tumor (TNM 7th), N (\%) } & $\mathrm{T} 1$ & $1 \quad(1.1)$ & & Paralytic ileus & $11(11.9)$ \\
\hline & $\mathrm{T} 2$ & $5 \quad(5.4)$ & & Remote infections & $2(2.2)$ \\
\hline & $\mathrm{T} 3$ & $63(68.5)$ & Length of hospital stay (day) & Median (range) & $11(4-51)$ \\
\hline & $\begin{array}{l}\text { T4a } \\
\text { T4b }\end{array}$ & $\begin{array}{rr}14 & (15.2) \\
9 & (9.8)\end{array}$ & \multirow{11}{*}{\multicolumn{3}{|c|}{$\begin{array}{l}\text { Remote infections were pneumonia and a urinary tract infection } \\
P S \text { Peformance status, ECOG Eastern Cooperative Oncology } \\
\text { Group, } \\
C \text { Cecum, } A \text { Ascending colon, } T \text { Transverse colon, } D \text { Descending } \\
\text { colon, } S \text { Sigmoid colon } \\
C E A \text { Carcinoembryonic antigen, } C A 19-9 \text { Carbohydrate antigen 19-9 } \\
H A R \text { High anterior resection, } L A R \text { Low anterior resection, } \\
u L A R \text { Ultra-low anterior resection, } A P R \text { Abdominoperineal } \\
\text { resection, } \\
L N \text { Lymph node, SSI Surgical site infection } \\
\text { a Japanese Classification of Colorectal Carcinoma, Second English } \\
\text { Edition (12) }\end{array}$}} \\
\hline \multirow[t]{2}{*}{ Lymphatic invasion, N (\%) } & Negative & $21(22.8)$ & & & \\
\hline & Positive & $71(77.2)$ & & & \\
\hline \multirow[t]{2}{*}{ Venous invasion, $\mathrm{N}(\%)$} & Negative & $15(16.3)$ & & & \\
\hline & Positiv & $77(83.7)$ & & & \\
\hline No. of LN metastases, $(\mathrm{N})$ & Median (range) & $0(0-7)$ & & & \\
\hline \multirow[t]{5}{*}{ LN metastasis (TNM 7th), N (\%) } & No & $52(56.5)$ & & & \\
\hline & N1a & $23(25.0)$ & & & \\
\hline & N1b & $13(14.1)$ & & & \\
\hline & $\mathrm{N} 2 \mathrm{a}$ & $3(3.3)$ & & & \\
\hline & $\mathrm{N} 2 \mathrm{~b}$ & $1 \quad(1.1)$ & & & \\
\hline \multirow[t]{6}{*}{ Stage (TNM7th), N (\%) } & II A & $43(46.7)$ & & & \\
\hline & II B & $4 \quad(4.4)$ & & & \\
\hline & II $\mathrm{C}$ & $5 \quad(5.4)$ & & & \\
\hline & III A & $12(13.0)$ & & & \\
\hline & III B & $22(23.9)$ & & & \\
\hline & III C & $6 \quad(6.5)$ & & & \\
\hline
\end{tabular}

developed distant recurrences (median time to relapse, 15.1 months) - three, three, two and one developed metastases of the liver, lungs, distant lymph nodes, and omentum, respectively. When recurrences were categorized according to stage (TNM7th), four patients $(23.5 \%)$ were in stage II A, two (11.8\%) were in II B, three (17.6\%) were in IIC, two (11.8\%) were in IIIA, four $(23.5 \%)$ were in IIIB, and two (11.8\%) were in IIIC.

As the first treatment for recurrence, eight patients underwent an operation, five patients received L-OHP-based adjuvant chemotherapy such as FOLFOX or CPT-11 plus infusional leucovorin and fluorouracil (FOLFIRI), three patients received S-1 plus CPT-11, and one patient received best supportive care. At last follow-up, five patients were still receiving chemotherapy. 
Table 2. Characteristics of patients with disease recurrence (median follow-up period, 70 months [range, 9-120 months])

\begin{tabular}{|c|c|c|c|c|c|c|c|c|c|}
\hline Case & Sex & $\begin{array}{c}\text { Age } \\
\text { (years) }\end{array}$ & Location & Operation & $\begin{array}{l}\text { Histologic } \\
\text { type }\end{array}$ & $\begin{array}{l}\text { Depth of } \\
\text { tumor }\end{array}$ & Stage & $\begin{array}{l}\text { No. of LNs } \\
\text { examined }(\mathrm{N})\end{array}$ & Site of recurrence \\
\hline 1 & Male & 71 & A & Elective & tubular & $\mathrm{T} 3$ & IIa & 10 & Lung \\
\hline 2 & Female & 52 & A & Emergency & tubular & $\mathrm{T} 4 \mathrm{~b}$ & IIc & 25 & Anastomosis \\
\hline 3 & Female & 55 & $\mathrm{~S}$ & Emergency & tubular & T4a & IIIb & 4 & Cervical lymph node \\
\hline 4 & Female & 70 & $\mathrm{~S}$ & Elective & tubular & $\mathrm{T} 4 \mathrm{~b}$ & IIIc & 8 & Liver \\
\hline 5 & Male & 76 & A & Elective & tubular & $\mathrm{T} 4 \mathrm{~b}$ & IIc & 41 & Anastomosis \\
\hline 6 & Female & 56 & $\mathrm{D}$ & Elective & tubular & $\mathrm{T} 3$ & IIa & 9 & Lung \\
\hline 7 & Male & 62 & $\mathrm{D}$ & Elective & tubular & $\mathrm{T} 3$ & IIa & 5 & Omentum \\
\hline 8 & Female & 80 & $\mathrm{C}$ & Elective & tubular & $\mathrm{T} 4 \mathrm{~b}$ & IIIc & 22 & Peritoneum \\
\hline 9 & Female & 77 & $\mathrm{RS}$ & Elective & tubular & $\mathrm{T} 3$ & IIIb & 17 & Liver \\
\hline 10 & Male & 73 & $\mathrm{D}$ & Elective & tubular & T4a & $\mathrm{IIb}$ & 11 & Anastomosis \\
\hline 11 & Female & 54 & $\mathrm{Rb}$ & Elective & tubular & $\mathrm{T} 3$ & IIa & 20 & Lung \\
\hline 12 & Male & 65 & $\mathrm{Ra}$ & Elective & tubular & T4a & IIIa & 12 & Peritoneum \\
\hline 13 & Male & 58 & $\mathrm{Rb}$ & Elective & tubular & $\mathrm{T} 3$ & IIIa & 28 & Anastomosis \\
\hline 14 & Male & 49 & $\mathrm{C}$ & Elective & mucinous & T4a & $\mathrm{IIb}$ & 50 & Mediastinal lymph node \\
\hline 15 & Male & 64 & $\mathrm{C}$ & Elective & tubular & T4a & IIIb & 24 & Liver \\
\hline 16 & Male & 73 & $\mathrm{~S}$ & Elective & tubular & $\mathrm{T} 4 \mathrm{~b}$ & IIc & 12 & Anastomosis \\
\hline 17 & Female & 57 & $S$ & Elective & tubular & T4a & IIIb & 19 & Peritoneum \\
\hline \multicolumn{7}{|c|}{ Treatment for recurrence } & $\begin{array}{l}\text { Time to } \\
\text { relapse } \\
\text { (M) }\end{array}$ & $\begin{array}{c}\text { Over all } \\
\text { Survival } \\
\text { (M) }\end{array}$ & Prognosis \\
\hline 1 & \multicolumn{6}{|c|}{ Operation $\rightarrow$ S-1 / CPT11 $\rightarrow$ Bev. / FOLFOX $\rightarrow$ Operation $\rightarrow$ Bev. / FOLFIRI $\rightarrow$ BSC } & 15.1 & 90 & Unknown \\
\hline 2 & \multicolumn{6}{|c|}{ FOLFOX $\rightarrow$ BSC } & 6.8 & 12 & Death \\
\hline 3 & \multicolumn{6}{|c|}{ Bev.FOLFIRI（Ongoing） } & 78 & 114 & Survival \\
\hline 4 & \multicolumn{6}{|c|}{ Operation $\rightarrow$ Bev. / FOLFIRI $\rightarrow$ BSC } & 6.8 & 53 & Death \\
\hline 5 & \multicolumn{6}{|c|}{ Operation $\rightarrow$ BSC } & 17 & 31 & Death \\
\hline 6 & \multicolumn{6}{|c|}{ Operation $\rightarrow$ Bev. / FOLFOX $\rightarrow$ BevFOLFIRI $\rightarrow$ Cetuximab（Ongoing） } & 36.5 & 98 & Survival \\
\hline 7 & \multicolumn{6}{|c|}{ Operation $\rightarrow$ Bev. / FOLFOX $\rightarrow$ Bev. / FOLFIRI $\rightarrow$ Cetuximab (Ongoing) } & 27.3 & 96 & Survival \\
\hline 8 & \multicolumn{6}{|c|}{ Not chemotherapy (BSC) } & 26.7 & 43 & Death \\
\hline 9 & \multicolumn{6}{|c|}{ Operation } & 8 & 50 & Unknown \\
\hline 10 & \multicolumn{6}{|c|}{ Operation $\rightarrow$ Lung metastasis $\rightarrow$ BSC } & 35.5 & 87 & Death \\
\hline 11 & \multicolumn{6}{|c|}{ S-1 / CPT-11 $\rightarrow$ Bev FOLFOX (Ongoing) } & 15.1 & 90 & Survival \\
\hline 12 & \multicolumn{6}{|c|}{ Bev. / FOLFOX $\rightarrow$ Bev. / FOLFORI $\rightarrow$ BSC } & 24.2 & 38 & Death \\
\hline 13 & \multicolumn{6}{|c|}{ FOLFOX $\rightarrow$ FOLFIRI } & 22.3 & 64 & Unknown \\
\hline 14 & \multicolumn{6}{|c|}{ S-1 / CPT-11 $\rightarrow$ Bev. / Xelox $\rightarrow$ Cetuximab / CPT-11 $\rightarrow$ BSC } & 12 & 38 & Death \\
\hline 15 & \multicolumn{6}{|c|}{ S-1 / CPT-11 $\rightarrow$ Bev. / FOLFOX $\rightarrow$ BSC } & 11 & 22 & Death \\
\hline 16 & \multicolumn{6}{|c|}{ Operation $\rightarrow$ Capecitabine (Ongoing) } & 22.1 & 80 & Survival \\
\hline 17 & \multicolumn{6}{|c|}{ Bev. / FOLFOX $\rightarrow$ BSC } & 26.3 & 53 & Unknown \\
\hline
\end{tabular}

$A$ Ascending colon, $S$ Sigmoid colon, $D$ Descending colon, $R S$ Rectosigmoid, $R a$ Rectum (above the peritoneal reflection), $R b$ Rectum (below the peritoneal reflection), M Months, FOLFOX Oxaliplatin plus infusional leucovorin and fluorouracil, FOLFIRI CPT-11 plus infusional leucovorin and fluorouracil, Bev. Bevacizumab, BSC Best supportive care

At the time of the final analysis, eight patients $(8.7 \%)$ had died due to disease progression. The 5-year DFS and OS rates for the entire study population were $82.2 \%$ and $91.9 \%$, respectively (Fig. 1a, b). 

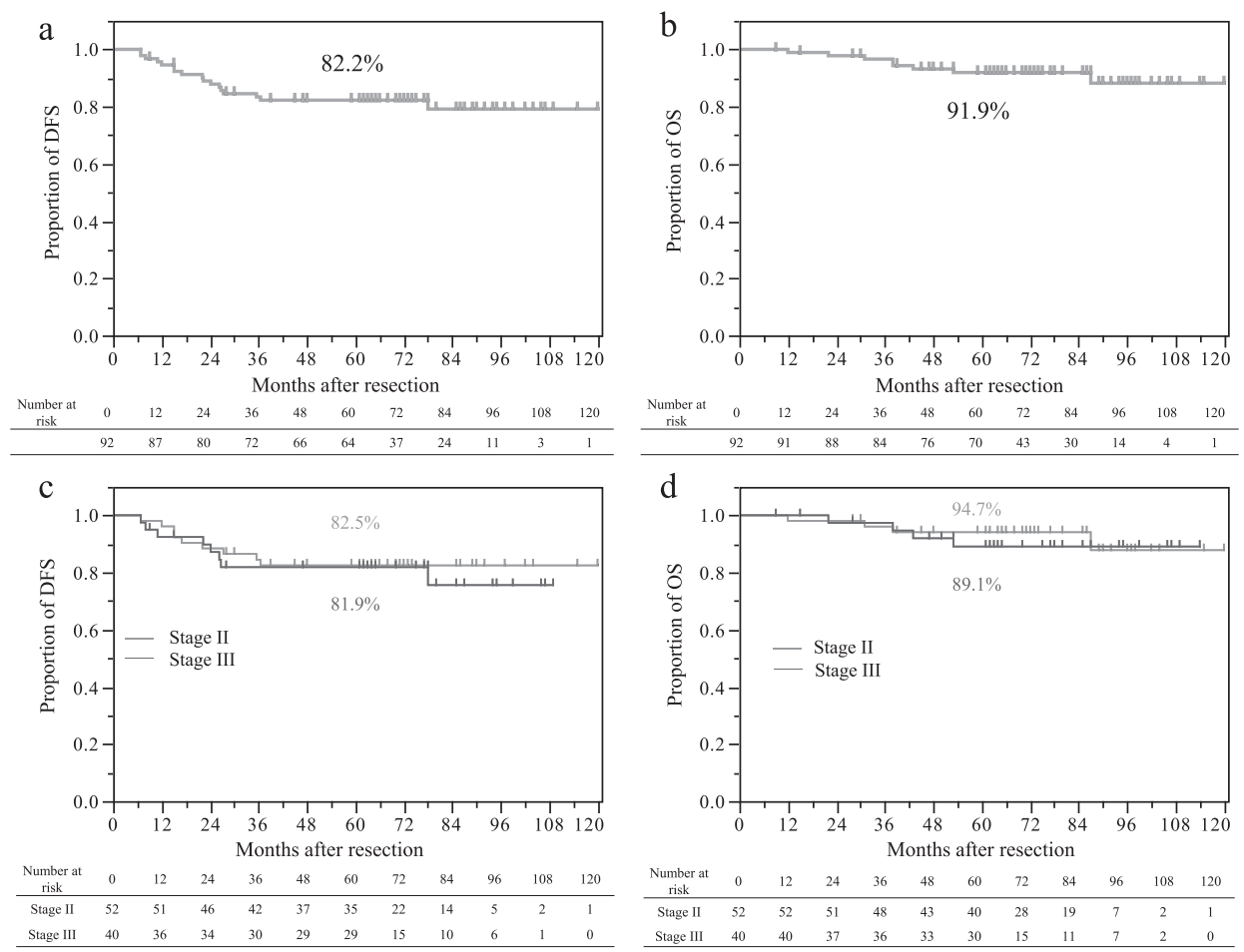

Fig. 1. Disease-free survival (DFS) and overall survival (OS) curves for all patients and for patients in Stage II and III.

(a) DFS curve for all patients; the 5-year DFS rate was $82.2 \%$.

(b) OS curve for all patients; the 5-year OS rate was $91.9 \%$.

(c) DFS curves for Stage II and Stage III patients; the 5-year DFS rates for Stage II and Stage III patients were $82.5 \%$ and $81.9 \%$, respectively. There was no significant difference in 5-year DFS between Stage II and Stage III patients (log-rank test; $p=$ $0.696)$.

(d) OS curves for Stage II and Stage III patients; the 5-year OS rates for Stage II and Stage III patients were $94.7 \%$ and $89.1 \%$, respectively. There was no significant difference in 5-year OS between Stage II and Stage III patients (log-rank test; $p=$ $0.674)$.

\section{Prognostic factors for recurrence}

Results of the univariate and multivariate analyses using the Cox proportional hazard model to identify significant prognostic factors for recurrence are presented in Table 3 . In the univariate analysis, five variables were identified as significant prognostic factors for recurrence affecting DFS : preoperative CA19-9 level > $37 \mathrm{U} / \mathrm{ml}$ (hazard ratio [HR], 6.520; 95\% CI, 1.961-19.650 ; $p=0.0036$ ), emergency operation (HR, 8.978; 95\% CI, 1.404-32.284; $p=0.025)$, D1 LN dissection (HR, 6.977; 95\% CI, 1.093-25.034; $p=0.042)$, T4 lesions (HR, 7.553; 95\% CI, 2.860-22.015; $p<0.0001$ ), and $>3 \mathrm{LN}$ metastases (HR, 5.661; 95\% CI, 1.296-17.570; $p=0.025$ ). In the multivariate analysis, preoperative CA19-9 level >37 U/ml (HR, 7.826; 95\% CI, 1.562-33.271; $p=0.016$ ), emergency operation (HR, 3.560e $+9 ; 95 \%$ CI, $1.323-1.164 \mathrm{e}+20 ; p=0.038)$, and T4 lesions (HR, 5.571; 95\% CI, 1.472-22.184; $p=0.012$ ) were independent significant prognostic factors after treatment. Both DFS and OS were significantly different according to the number of independent prognostic factors for recurrence (number of prognostic factors 0 vs $\geq 1$ : HR, 
Table 3. Univariate and multivariate analyses using the Cox proportional hazard model to identify significant prognostic factors for recurrence

\begin{tabular}{|c|c|c|c|c|c|}
\hline \multirow[b]{2}{*}{ Factor } & \multicolumn{3}{|c|}{ Univariate analysis } & \multicolumn{2}{|c|}{ Multivariate analysis } \\
\hline & Hazard & ratio $(95 \% \mathrm{CI})$ & $P$ value & Hazard ratio $(95 \% \mathrm{CI})$ & $P$ value \\
\hline \multicolumn{6}{|l|}{ Age (years) } \\
\hline$>65$ & 0.639 & $(0.232-1.667)$ & 0.36 & & \\
\hline$\leq 65$ & & 1 & & & \\
\hline \multicolumn{6}{|l|}{ Sex } \\
\hline Male & 0.584 & $(0.223-1.556)$ & 0.274 & & \\
\hline Female & & 1 & & & \\
\hline \multicolumn{6}{|c|}{ Preoperative CEA level (ng/ml) } \\
\hline$>5.1$ & 1.312 & $(0.422-3.952)$ & 0.627 & & \\
\hline$\leq 5.1$ & & 1 & & & \\
\hline \multicolumn{6}{|c|}{ Preoperative CA19-9 level（U/ml） } \\
\hline$>37$ & 6.520 & $(1.961-19.650)$ & 0.0036 & $11.038(2.517-47.262)$ & 0.0025 \\
\hline$\leq 37$ & & 1 & & 1 & \\
\hline \multicolumn{6}{|l|}{ Operation } \\
\hline Emergency & 8.978 & $(1.404-32.284)$ & 0.025 & $15.002(1.732-101.219)$ & 0.017 \\
\hline Elective & & 1 & & 1 & \\
\hline \multicolumn{6}{|l|}{ Tumor location } \\
\hline Colon & 2.561 & $(0.905-9.102)$ & 0.078 & & \\
\hline Rectum & & 1 & & & \\
\hline \multicolumn{6}{|l|}{ Surgical approach } \\
\hline Laparotomy & 1.792 & $(0.663-6.640)$ & 0.258 & & \\
\hline Laparoscopic & & 1 & & & \\
\hline \multicolumn{6}{|l|}{ Tumor size } \\
\hline$>50$ & 0.783 & $(0.284-2.040)$ & 0.618 & & \\
\hline$\leq 50$ & & 1 & & & \\
\hline \multicolumn{6}{|l|}{ LN dissection } \\
\hline D1 & 6.977 & $(1.093-25.034)$ & 0.042 & $2.058(0.088-23.763)$ & 0.592 \\
\hline D2, D3 & & 1 & & 1 & \\
\hline \multicolumn{6}{|l|}{ Histologic types } \\
\hline Mucinous, poorly & 1.695 & $(0.266-6.070)$ & 0.513 & & \\
\hline Tubular, Papirally & & 1 & & & \\
\hline \multicolumn{6}{|l|}{ Depth of tumor } \\
\hline $\mathrm{T} 4$ & 7.553 & $(2.860-22.015)$ & $<0.0001$ & $4.956(1.172-21.230)$ & 0.03 \\
\hline$\leq \mathrm{T} 3$ & & 1 & & 1 & \\
\hline \multicolumn{6}{|l|}{ Lymphatic invasion } \\
\hline Positive & 1.053 & $(0.297-2.977)$ & 0.928 & & \\
\hline Negative & & 1 & & & \\
\hline \multicolumn{6}{|l|}{ Venous invasion } \\
\hline Positive & 1.539 & $(0.434-9.767)$ & 0.545 & & \\
\hline Negative & & 1 & & & \\
\hline \multicolumn{6}{|l|}{ No. of LNs examined } \\
\hline$>13$ & 1.952 & $(0.733-5.107)$ & 0.175 & & \\
\hline$\leq 13$ & & 1 & & & \\
\hline \multicolumn{6}{|l|}{ No. of LN metastases } \\
\hline$>3$ & 5.661 & $(1.296-17.570)$ & 0.025 & $4.101 \quad(0.572-19.610)$ & 0.14 \\
\hline$\leq 3$ & & 1 & & 1 & \\
\hline \multicolumn{6}{|l|}{ Stage } \\
\hline II & 1.208 & $(0.453-3.162)$ & 0.698 & & \\
\hline III & & 1 & & & \\
\hline \multicolumn{6}{|c|}{ Postoperative complications } \\
\hline+ & 1.948 & $(0.676-5.131)$ & 0.207 & & \\
\hline- & & 1 & & & \\
\hline
\end{tabular}

CEA Carcinoembryonic antigen, CA19-9 Carbohydrate antigen 19-9 

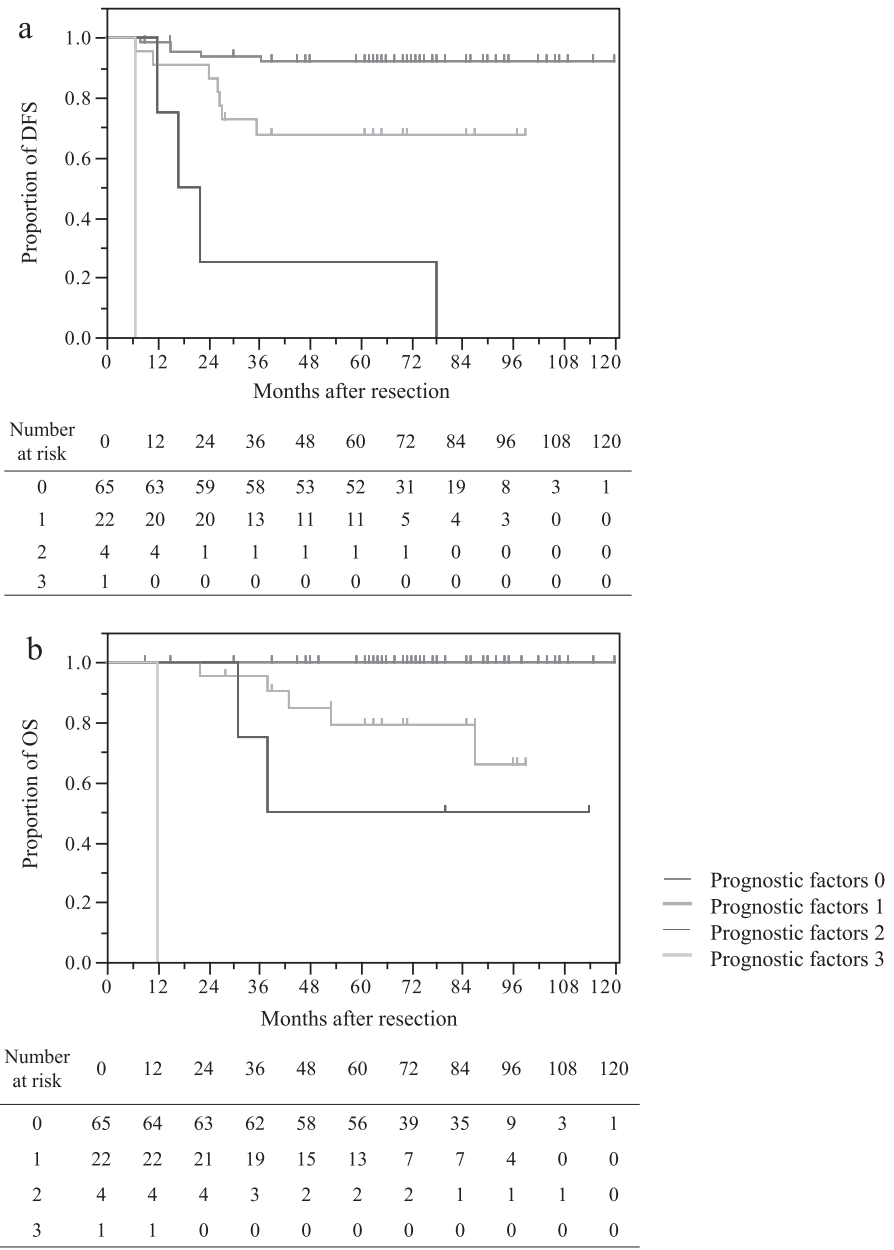

Fig. 2. Disease-free survival (DFS) and overall survival (OS) curves based on the number of prognostic factors for recurrence. $^{*}$

(a) DFS curves based on the number of prognostic factors for recurrence

(b) OS curves based on the number of prognostic factors for recurrence

* Number of prognostic factors 0 vs $\geq 1$ : hazard ratio, 6.976; 95\% CI, 2.580-21.973; log-rank test, $p<0.0001$; Wilcoxon test, $p<0.0001$.

6.976; 95\% CI, 2.580-21.973; log-rank test, $p<0.0001$; Wilcoxon test, $p<0.0001$ ) (Fig. 2).

\section{Discussion}

To our knowledge, this is the first report on the analysis of prognostic factors for recurrence after UFT / LV adjuvant chemotherapy for patients with CRC. In this study, common sites of recurrence after UFT/LV adjuvant chemotherapy included the anastomosis (29.4\% [5/17]), and other local sites, the liver and the lungs (17.6\% [3/17] each). According to the JSCCR, the recurrence rates after curative resection are $2.4 \%$ for the anastomosis, $23.1 \%$ for other local 
sites, $41.2 \%$ for the liver, $27.6 \%$ for the lungs, and $22.0 \%$ for other sites (including overlaps) ${ }^{6}$. Although it is not possible to directly compare recurrence patterns, they may have been affected by UFT/LV adjuvant chemotherapy; in particular, hematogenous metastases such as liver and lung metastases may have been suppressed by UFT/LV.

In NSABP C-06, which evaluated the non-inferiority of UFT / LV compared with intravenous 5-FU/ levofolinate (1-LV) as adjuvant chemotherapy in Stage II and III colon cancer, the 5-year DFS rate was $67.0 \%{ }^{3)}$. Conversely, in JCOG0205, which also evaluated the non-inferiority of UFT / LV compared with 5FU/1-LV in Stage III CRC, the 5-year DFS rate was 73.6\%. In our study, the 5-year DFS rate was $82.2 \%$ for all patients and $81.9 \%$ for patients in Stage III (Fig. 1c) ${ }^{2}$. The 5-year OS rate for patients in Stage III in our study was $89.1 \%$ (Fig. 1d), which was similar to the $87.5 \%$ in the JCOG0205 trial. In our study, 16 of the 17 patients who had recurrence underwent surgery or systemic chemotherapy for recurrence, and five received L-OHP- or 5FU-based chemotherapy during progression-free survival. Thus, in addition to the UFT / LV adjuvant chemotherapy, surgery and novel anticancer agents may have contributed to the remarkable improvement in the 5-year OS.

The pathological stage of T4 and intestinal perforation can identify a minority of Stage II CRC patients who have a higher risk of recurrence ${ }^{8-10)}$. Of the 17 patients who had recurrence after UFT / LV adjuvant chemotherapy in our study, nine were in Stage II (Table 2). Of them, five had T4 lesions, and three had $<13$ sampled LNs. This meant that eight of the nine Stage II patients who had recurrence $(88.9 \%)$ had high-risk Stage II disease. Therefore, aggressive adjuvant chemotherapy should given to patients with CRC who are in Stage III and to a minority of those in high-risk Stage II.

Serum CEA levels have been shown to be elevated in a majority of patients with recurrence after curative resection for $\mathrm{CRC}^{14)}$. In particular, $80 \%$ of patients with hepatic recurrence had elevated serum CEA levels. In this study, the preoperative CEA level was not a significant prognostic factor for recurrence after UFT/LV adjuvant chemotherapy in the univariate analysis. The UFT/LV adjuvant chemotherapy may have suppressed hematogenous metastases such as liver and lung metastases; as a result, preoperative CEA level was not selected as a prognostic factor for recurrence. Although the American Society of Clinical Oncology guidelines for the use of tumor markers in CRC state that there are insufficient data to recommend CA19-9 for screening, diagnosis, staging, surveillance, or treatment monitoring in $\mathrm{CRC}^{15}$, CA19-9 has been widely used as a tumor marker for CRC. Takakura et al reported that the preoperative CA199 level was a significant predictor of peritoneal dissemination and poor survival in patients with $\mathrm{CRC}^{16)}$. Furthermore, Nakagoe et al reported that the preoperative CA19-9 level might serve as a useful marker in identifying node-negative CRC patients who are at high risk of recurrence after surgery ${ }^{17)}$. Since recurrence patterns may have changed with UFT/LV adjuvant chemotherapy and the rate of peritoneal recurrence was high, we selected the preoperative CA19-9 level (which is a significant predictor of peritoneum recurrence) as an independent prognostic factor for recurrence.

The 5-year DFS and OS rates for patients who had at least one of the independent 
prognostic factors (T4 lesions, emergency operation, and high preoperative CA19-9 level) were significantly worse than those for patients who did not have any of these three factors. Therefore, Stage III or high-risk Stage II CRC patients with none of the three independent prognostic factors may be suitable for oral 5-FU-based adjuvant chemotherapy. Stage III or highrisk Stage II patients who have any one of three independent prognostic factors should receive another recommended adjuvant therapy, such as L-OHP-based chemotherapy, because they have a very high likelihood of recurrence.

Several limitations to our study must be considered. First, the number of patients was small. This meant that while emergency operation was selected as an independent prognostic factor, only two patients had emergency operations in our study. Therefore, type I errors could not be avoided when analyzing prognostic factors for recurrence. However, CRC with perforation was not found to be an independent prognostic factor in our study, although it has previously been shown to be a risk factor for recurrence and to indicate poor prognosis ${ }^{18-20)}$, so it must be an important prognostic factor. Second, we conducted a retrospective study at a single institution, not a prospective study that compared CRC patients with and without adjuvant therapy. Third, our study did not include the biomarkers associated with CRC recurrence or molecular markers associated with response to anticancer agents. Several molecular markers have been shown to be satisfactory predictors of the efficacy of 5-FU-based or L-OHP-based chemotherapy ${ }^{21-27)}$. Yothers et al reported that the 12-gene Recurrence Score provides additional information beyond the conventional clinical and pathological factors ${ }^{28)}$; this score is a predictor of recurrence risk that was developed using gene expression data ${ }^{29)}$. In clinical practice, these factors could improve decision-making regarding adjuvant chemotherapy for patients with Stage II and III colon cancer.

In conclusion, for patients with CRC who receive UFT/LV adjuvant chemotherapy, three independent prognostic factors - T4 lesions, emergency operation, and a high preoperative CA199 level - may be useful for decision-making regarding the choice between 5-FU-based and L-OHP-based adjuvant chemotherapy.

\section{Conflict of interest disclosure}

We have no conflicts of interest to declare.

\section{References}

1) Chau I, Cunningham D. Adjuvant therapy in colon cancer - what, when and how? Ann Oncol. 2006;17:13471359.

2) Shimada Y, Hamaguchi T, Mizusawa J, et al. Randomised phase III trial of adjuvant chemotherapy with oral uracil and tegafur plus leucovorin versus intravenous fluorouracil and levofolinate in patients with stage III colorectal cancer who have undergone Japanese D2/D3 lymph node dissection: final results of JCOG0205. Eur J Cancer. 2014;50:2231-2240.

3) Lembersky BC, Wieand HS, Petrelli NJ, et al. Oral uracil and tegafur plus leucovorin compared with intravenous fluorouracil and leucovorin in stage II and III carcinoma of the colon: results from National Surgical Adjuvant 
Breast and Bowel Project Protocol C-06. J Clin Oncol. 2006;24:2059-2064.

4) Andre T, Boni C, Mounedji-Boudiaf L, et al. Oxaliplatin, fluorouracil, and leucovorin as adjuvant treatment for colon cancer. N Engl J Med. 2004;350:2343-2351.

5) Twelves C, Wong A, Nowacki MP, et al. Capecitabine as adjuvant treatment for stage III colon cancer. $N$ Engl $J$ Med. 2005;352:2696-2704.

6) Watanabe T, Itabashi M, Shimada Y, et al. Japanese Society for Cancer of the Colon and Rectum. Japanese Society for Cancer of the Colon and Rectum (JSCCR) guidelines 2010 for the treatment of colorectal cancer. Int J Clin Oncol. 2012;17:1-29.

7) Yoshida M, Ishiguro M, Ikejiri K, et al. ACTS-CC Study Group. S-1 as adjuvant chemotherapy for stage III colon cancer: a randomized phase III study (ACTS-CC trial). Ann Oncol. 2014;25:1743-1749.

8) Benson AB 3rd, Schrag D, Somerfield MR, et al. American Society of Clinical Oncology recommendations on adjuvant chemotherapy for stage II colon cancer. J Clin Oncol. 2004;22:3408-3419.

9) Quasar Collaborative Group; Gray R, Barnwell J, McConkey C, et al. Adjuvant chemotherapy versus observation in patients with colorectal cancer: a randomised study. Lancet. 2007;370:2020-2029.

10) Engstrom PF, Arnoletti JP, Benson AB 3rd, et al. National Comprehensive Cancer Network. NCCN Clinical Practice Guidelines in Oncology: colon cancer. J Natl Compr Canc Netw. 2009;7:778-831.

11) Tsunoda A, Nakao K, Watanabe $\mathrm{M}$, et al. Associations of various gene polymorphisms with toxicity in colorectal cancer patients receiving oral uracil and tegafur plus leucovorin: a prospective study. Ann Oncol. 2011;22:355-361.

12) Japanese Society for Cancer of the Colon and Rectum. Japanese classification of colorectal carcinoma. 2nd English ed. Tokyo: Kanehara; 2009.

13) Sobin LH, Gospodarowicz MK, Wittekind C. UICC International Union Against Cancer, TNM classification of malignant tumors. 7th ed. New York: Wiley-Blackwell; 2010.

14) McCall JL, Black RB, Rich CA, et al. The value of serum carcinoembryonic antigen in predicting recurrent disease following curative resection of colorectal cancer. Dis Colon Rectum. 1994;37:875-881.

15) Locker GY, Hamilton S, Harris J, et al. ASCO. ASCO 2006 update of recommendations for the use of tumor markers in gastrointestinal cancer. J Clin Oncol. 2006;24:5313-5327.

16) Takakura Y, Ikeda S, Imaoka Y, et al. An elevated preoperative serum carbohydrate antigen 19-9 level is a significant predictor for peritoneal dissemination and poor survival in colorectal cancer. Colorectal Dis. 2015;17:417-425.

17) Nakagoe T, Sawai T, Tsuji T, et al. Preoperative serum level of CA19-9 predicts recurrence after curative surgery in node-negative colorectal cancer patients. Hepatogastroenterology. 2003;50:696-699.

18) Abdelrazeq AS, Scott N, Thorn C, et al. The impact of spontaneous tumour perforation on outcome following colon cancer surgery. Colorectal Dis. 2008;10:775-780.

19) Khan S, Pawlak SE, Eggenberger JC, et al. Acute colonic perforation associated with colorectal cancer. Am Surg. 2001;67:261-264.

20) Carraro PG, Segala M, Orlotti C, et al. Outcome of large-bowel perforation in patients with colorectal cancer. Dis Colon Rectum. 1998;41:1421-1426.

21) Mekata E, Sonoda H, Shimizu $\mathrm{T}$, et al. Clinical predictive value of in vitro anticancer drug sensitivity test for the therapeutic effect of adjuvant chemotherapy in patients with stage II-III colorectal cancer. Mol Clin Oncol. 2013;1:763-767.

22) Watanabe T, Wu TT, Catalano PJ, et al. Molecular predictors of survival after adjuvant chemotherapy for colon cancer. N Engl J Med. 2001;344:1196-1206.

23) Sargent DJ, Marsoni S, Monges G, et al. Defective mismatch repair as a predictive marker for lack of efficacy of fluorouracil-based adjuvant therapy in colon cancer. J Clin Oncol. 2010;28:3219-3226. Erratum in: J Clin Oncol. 2010;28:4664.

24) Sinicrope FA, Foster NR, Thibodeau SN, et al. DNA mismatch repair status and colon cancer recurrence and 
survival in clinical trials of 5-fluorouracil-based adjuvant therapy. J Natl Cancer Inst. 2011;103:863-875. Erratum in: J Natl Cancer Inst. 2011;103:1639.

25) Farina Sarasqueta A, van Lijnschoten G, Lemmens VE, et al. Pharmacogenetics of oxaliplatin as adjuvant treatment in colon carcinoma: are single nucleotide polymorphisms in GSTP1, ERCC1, and ERCC2 good predictive markers? Mol Diagn Ther. 2011;15:277-283.

26) Harradine KA, Kassner M, Chow D, et al. Functional genomics reveals diverse cellular processes that modulate tumor cell response to oxaliplatin. Mol Cancer Res. 2011;9:173-182.

27) Shirota Y, Stoehlmacher J, Brabender J, et al. ERCC1 and thymidylate synthase mRNA levels predict survival for colorectal cancer patients receiving combination oxaliplatin and fluorouracil chemotherapy. J Clin Oncol. 2001;19:4298-4304.

28) Yothers G, O'Connell MJ, Lee M, et al. Validation of the 12-gene colon cancer recurrence score in NSABP C-07 as a predictor of recurrence in patients with stage II and III colon cancer treated with fluorouracil and leucovorin (FU / LV) and FU/LV plus oxaliplatin. J Clin Oncol. 2013;31:4512-4519. Erratum in: J Clin Oncol. 2014;32:866.

29) O’Connell MJ, Lavery I, Yothers G, et al. Relationship between tumor gene expression and recurrence in four independent studies of patients with stage II / III colon cancer treated with surgery alone or surgery plus adjuvant fluorouracil plus leucovorin. J Clin Oncol. 2010;28:3937-3944.

[Received October 19, 2015 : Accepted November 4, 2015] 